British Journal of Nutrition (2022), 128, 2258-2266

doi: $10.1017 / \mathrm{S} 0007114522000265$

(C) The Author(s), 2022. Published by Cambridge University Press on behalf of The Nutrition Society. This is an Open Access article, distributed under the terms of the Creative Commons Attribution-NonCommercial-NoDerivatives licence (http://creativecommons.org/licenses/by-nc-nd/4.0/), which permits non-commercial re-use, distribution, and reproduction in any medium, provided that no alterations are made and the original article is properly cited. The written permission of Cambridge University Press must be obtained prior to any commercial use and/or adaptation of the article.

\title{
Dietary inflammation index and osteoarthritis in the elderly: is there a mediating role of physical activity?
}

\author{
Haoyi Wang ${ }^{1} \dagger$, Runzhi Liao ${ }^{1} \dagger$, Weifeng Tang ${ }^{2}$, Weiping Su${ }^{1}$, Min Zeng ${ }^{1}$, Junxiao Yang ${ }^{1}$, Xiaolei Fan ${ }^{1}$, \\ Jie $\mathrm{Xie}^{1,3 *}$ and Yihe $\mathrm{Hu}^{1,3,4,5 *}$ \\ ${ }^{1}$ Department of Orthopedics, Xiangya Hospital, Central South University, Changsha, Hunan, People's Republic of China \\ ${ }^{2} X i n h u a$ Hospital, Shanghai Jiao Tong University, School of Medicine, Shanghai, People's Republic of China \\ ${ }^{3}$ Hunan Engineering Research Center of Biomedical Metal and Ceramic Implants, Xiangya Hospital, Central South University, \\ Changsha, Hunan, People's Republic of China \\ ${ }^{4}$ Department of Orthopedic Surgery, The First Affiliated Hospital, College of Medicine, Zhejiang University, Hangzhou, People's \\ Republic of China \\ ${ }^{5}$ State Key Laboratory of Powder Metallurgy, Central South University, Changsha, People's Republic of China
}

(Submitted 10 November 2021 - Final revision received 4 January 2022 - Accepted 19 January 2022 - First published online 24 January 2022 )

\section{Abstract}

We examined whether physical activity (PA) explains the association between dietary inflammatory potential and osteoarthritis (OA) in the elderly. A total of 1249 elderly people ( $\geq 65$ years) were eligible for this study from the National Health and Nutrition Examination Survey (NHANES) from 2011 to 2016. The semi-quantitative Food Frequency Questionnaire (FFQ) and Global PA Questionnaire (GPAQ) were used to evaluate the diet and PA of the elderly, respectively. The multivariable logistic regression model estimated the odds ratio (OR) and 95\% confidence interval (CI) between Energy-adjusted Dietary Inflammatory Index (E-DII) and OA. The interaction of E-DII and PA on depressive events was tested, and the mediation analysis of PA was performed. The average E-DII in this study was +0.68 (SE 0.08 ), and the score ranges from -5.32 (most anti-inflammatory) to +4.26 (most pro-inflammatory). In comparison with the first quartile, the elderly from the second quartile (OR: 1.16 [95\% CI: 1.06, 1.68]) to the fourth quartile (OR: 1.64 [95\% CI: 1.13, 2.37]) had a higher risk of OA before adjustment for PA. An interaction was observed between E-DII and PA in terms of the risk of OA $\left(P_{\text {Interaction }}<0.001\right)$. The whole related part was mediated by PA $(20.08 \%)$. Our findings indicated that the higher pro-inflammatory potential of diet was associated with a higher risk of OA, and low PA was an important part of the mediating factor in the relationship between systemic low-grade dietary inflammation and the risk of OA.

Keywords: Dietary inflammatory index: Osteoarthritis: Physical activity: Mediation analysis

Osteoarthritis (OA) is the most common degenerative joint disease in the USA and the fastest cause of disability and pain. The prevalence of OA among American adults 20 years and older had increased from $6 \cdot 6 \%$ in 1999 to $14.3 \%$ in $2014^{(1)}$. The number of OA cases in the USA is expected to increase partly due to the obesity epidemic and the ageing population. Globally, approximately $18 \%$ of women aged over 60 and $9.6 \%$ of men have symptomatic OA. In addition, OA has been estimated to affect 130 million people by $2050^{(2)}$

Inflammation has been reported to play a potential role in the development of $\mathrm{OA}^{(3)}$. This role is already recognised through several features. For example, synovitis (defined as the inflammation of the synovium) is a common early symptom in patients with OA of the joint ${ }^{(4)}$. Epidemiological studies have shown that serum C-reactive protein levels are significantly associated with the incidence and progression of $\mathrm{OA}^{(5)}$. Other studies have demonstrated a positive association between the serum levels of C-reactive protein and the histological evidence of synovitis before joint replacement ${ }^{(6-8)}$. These observations strongly suggest that the systemic inflammation observed in OA reflects, at least in part, local synovial inflammation.

The dietary inflammation index (DII) is a literature-derived dietary tool that is used to assess the overall inflammatory potential of an individual's diet ${ }^{(9)}$. The association of high DII values

Abbreviations: DII, dietary inflammation index; MET, metabolic equivalents of task; NHANES, National Health and Nutrition Examination Survey; OA, osteoarthritis; PA, physical activity.

*Corresponding authors: Dr Y. Hu, email xy_huyh@163.com; Dr J. Xie, email dr_xiejie@163.com

$\dagger$ Haoyi Wang and Runzhi Liao contributed equally to the work and should be considered as first authors. 
with high serum IL- 6 and TNF- $\alpha$ levels suggests a strong relationship between this index and inflammatory parameters. DII is also used to assess the association of dietary inflammation with metabolic syndrome, asthma, breast cancer, colorectal cancer and fractures ${ }^{(10-13)}$. However, to our knowledge, no study has explored the relationship between DII and OA

Physical activity (PA) is related to the regulation of the innate immune system and the treatment of $\mathrm{OA}^{(14)}$. For example, regular PA may increase the body's serotonin synthesis, improve noradrenergic neurotransmission, trigger the release of endorphins and reduce the long-term increased sympathetic nervous system activity, thereby reducing systemic inflammation in the elderly ${ }^{(15)}$. In addition, individuals who regularly engage in PA are less likely to develop symptoms of OA in the future than those who do not. The possible mechanism is PA can prevent inflammation of chondrocytes and cartilage matrix by reducing IL- 6 and TNF- $\alpha$ and other OA-related inflammatory markers, delaying the degeneration of articular cartilage, thereby improving joint function. At the same time, it may increase the level of protective inflammation markers in the body to inhibit the effect of OA progression ${ }^{(16,17)}$. Therefore, regular PA may play a central role in maintaining physical and mental health. However, until now, no studies have examined whether PA acts as an intermediate mechanism in the relationship between low-grade systemic dietary inflammation and subsequent $\mathrm{OA}$.

Therefore, on the basis of the data from the National Health and Nutrition Examination Survey (NHANES), the present study aims to assess the association between E-DII and OA prospectively and to evaluate the potential interactions and the mediating role of PA in this relationship.

\section{Methods}

\section{Study population}

In the present study, we analysed the data collected from NHANES. NHANES is a cross-sectional study designed to assess the health and nutritional status of adults and children in the USA, which is conducted by the Centers for Disease Control and Prevention. The data were collected through questionnaire surveys, physical examinations, household interviews including demographic, socio-economic, dietary, health-related questions and examinations, and laboratory tests. Detailed information about survey design and methodologies can be found elsewhere $^{(18)}$. In this study, a total of 1249 participants aged 65 years were selected from the 2011-2016 NHANES (Fig. 1).

\section{Energy-Adjusted Dietary Inflammatory Index}

There are some studies on the development and verification of DII in detail ${ }^{(19)}$. Research from more than 1900 peer-reviewed publications forms the basis of DII. The 'Inflammatory Effect Score' was created from these peer-reviewed publications for each DII food parameter, based on their impact on inflammatory cytokines. The calculation of the dietary inflammatory index links the personal dietary data obtained in each clinical study with the global average intake ${ }^{(19)}$. The world database provides standard averages and deviations of all DII food parameters. For each food parameter, create a $Z$-score by subtracting the individual's estimated intake from the standard average. It is then divided by the world standard deviation and converted to a distribution centred at 0 and bounded between -1 and +1 . This value is then multiplied by the inflammatory effect score for each food parameter, and then all food parameters are added together to create an overall DII score. The more positive scores mean the more pro-inflammatory diet, the more negative values, the stronger the anti-inflammatory effect ${ }^{(20)}$.

DII calculation formula:

$\mathrm{Z}$ score $=(($ daily mean intake - global daily mean intake $) /$ standard deviation $)$

$Z$ score $^{1}=Z$ score $\rightarrow($ converted to a percentile score $) \times 2-1$

$\mathrm{DII}=\sum Z$ score $^{1} \times$ the inflammatory effect score of each dietary component

In this study, twenty-seven of the forty-five food parameters can be obtained through NHANES data, including grams of alcohol, protein, fibre, fat, carbohydrates, cholesterol, $n-3$ and $n-6$ polyunsaturated fatty acids, saturated fatty acids/MUFA/PUFA, Mg, niacin, $\mathrm{Zn}, \mathrm{Fe}$, riboflavin, folic acid, $\beta$-carotene, caffeine, Se, thiamine and vitamins $\mathrm{A}, \mathrm{B}_{6}, \mathrm{~B}_{12}, \mathrm{C}, \mathrm{D}$ and $\mathrm{E}$. DII scores range from negative tail to positive tail, more negative values indicate antiinflammatory properties and corrected scores indicate proinflammatory properties. E-DII represents the energy-adjusted amount of DII per 1000 energies in food intake to explain the impact of total intake on energy intake.

\section{Physical activity}

In NHANES, Participants self-reported their activity patterns through questions derived from the Global Physical Activity Questionnaire $^{(21)}$. Each participant was required to recall the type, frequency (exercise days per week) and duration (exercise times/d) of PA they had undertaken during the past $7 \mathrm{~d}$ for a minimum of $10 \mathrm{~min}$, including moderate and vigorous intensity related to work, transport and recreational activities.

Moderate/vigorous intensity activities are defined as activities that require moderate/hard physical effort and cause small/large increases in breathing or heart rate. Work-related physical activities are described as paid or unpaid work, household chores and yard work. For recreational PA, the information referred to sports, fitness and recreational activities. The active transportation domain referred to all walking and bicycling activities.

The metabolic equivalents of task (MET) are a unit used to describe the energy expenditure of a specific activity, where 1 MET refers to $3.5 \mathrm{ml} \mathrm{O}_{2} \mathrm{~kg}^{-1} / \mathrm{min}^{(21)}$. The suggested MET is 8.0 for vigorous work-related activity and vigorous leisure-time PA, while for moderate work-related activity, walking or bicycling for transportation and moderate leisure-time PA is $4 \cdot 0^{(22)}$. Each PA is assessed by MET-min/week by multiplying the days spent performing this activity by the mean duration by the suggested MET and sum each activity value to obtain an estimate of total PA. 
29902 Participants included in the NHANES (2011-2016)

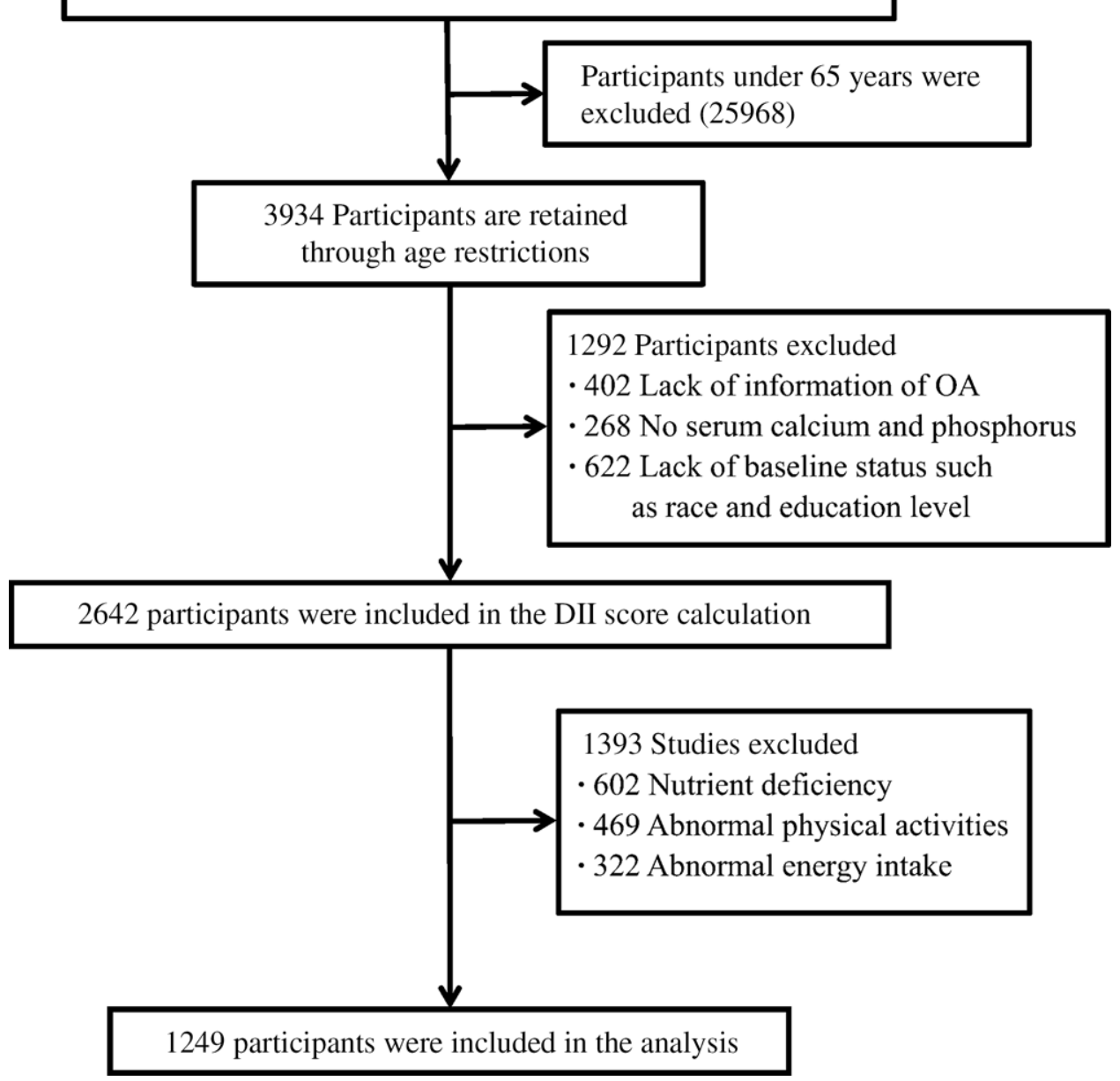

Fig. 1. Selection process of subjects.

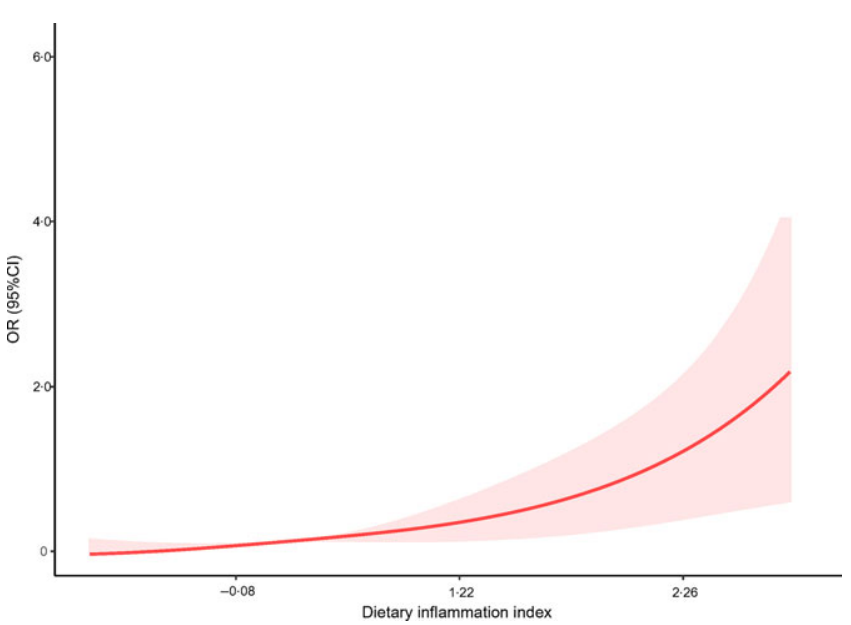

Fig. 2. A restricted cubic spline curve was used to fit the relationship between Energy-Adjusted Dietary Inflammatory Index (E-DII) and the risk of osteoarthritis (OA) (the three nodes are located at the 25th, 50th and 75th percentiles). Risk estimates were adjusted for age, sex, BMI, education level, race, hypertension, diabetes, marital status, poverty:income ratio (PIR), serum calcium and phosphorus. The reference value for OR: median score (1.22); the solid black line represents the OR and the shaded part the lower and upper $95 \% \mathrm{Cl}$; $P_{\text {Overall }}<0.001 ; P_{\text {Nonlinear }}=0.012$.

\section{Osteoarthritis status}

Information regarding the assessment of OA was obtained by selfreport questionnaire. A previous study demonstrated great consistency ( $85 \%$ ) between self-reported OA and clinically confirmed $\mathrm{OA}^{(23)}$. Participants were asked, 'Has a doctor or other health professional ever told you that you had arthritis?' If the answer was yes, a follow-up question was 'Which type of arthritis was it?' The participants were classified as OA groups based on the answers to the latter question. Those who did not have or had any signs or symptoms of arthritis were placed in the no arthritis group.

\section{Study covariates}

According to previous research, the multivariable model contains latent variables that confuse the association between E-DII and OA, which include age (continuous), race/ethnicity (category), BMI category, poverty:income ratio (continuous), hypertension (category), diabetes (category) and serum Ca (continuous) and serum phosphorus (continuous). NHANES calculated the BMI categories defined as four levels: underweight (BMI < 18.5), normal weight $(18 \cdot 5 \leq \mathrm{BMI}<24 \cdot 0)$, overweight $(24 \cdot 0 \leq \mathrm{BMI}<28 \cdot 0)$ and obesity (BMI $\geq 28 \cdot 0)$. Poverty: 
income ratio is calculated by dividing the family income (based on the poverty criteria specific to the size of the family) by the year and state and is used to embody the socio-economic status of the participant in the entire family. Race/ethnicity is coded as non-Hispanic white, non-Hispanic black, Hispanic (Mexican American and other Hispanics), etc.

\section{Statistical analyses}

The baseline characteristics of the study population were reported as the median (interquartile range, IQR) of continuous variables and the number (percentage) of categorical variables. We used a logistic proportional hazard model to estimate OR and $95 \%$ CI.

DII is modelled in three different ways. For our main analysis, E-DII was included as a continuous variable, and we used an estimate of the effect of the 1-SD increase ( $Z$-score) in E-DII. This method assumes a linear relationship between E-DII and OA. To verify the hypothesis, we then used a multivariable restricted cubic spline and placed three nodes at the 25th, 50th and 75th percentiles of the E-DII distribution node to provide a graphical representation. The spline curve allows us to evaluate whether there was a significant difference from the linear correlation. Finally, we divided E-DII into quarters and used the first quarter group as the reference category in the logical model.

Three models were proposed: the first model did not adjust for confounding factors; the second model further adjusted for known risk factors for depression and potential confounding factors, such as age, gender, BMI, education level, marital status, race, hypertension, diabetes, serum $\mathrm{Ca}$ and phosphorus concentrations; and the third model further adjusted PA. Confounding factors were pre-selected based on previously published articles and were associated with OA and E-DII ${ }^{(24,25)}$

The interaction of E-DII and PA on the risk of OA was estimated by including a multiplication term between the two variables in the logistic model. Considering statistical significance, we examined the association between E-DII and OA stratified by quartile based on PA.

Regression-based mediation analysis was used to distinguish the direct effect of adherence to E-DII on the risk of depression and the indirect effect mediated by BMI. Three estimates were obtained as follows:

- Total effect, that is, the overall association between E-DII and the risk of depression, including the association mediated by BMI.

- Direct effect, that is, the association between E-DII and depression risk, adjusted according to BMI.

- Indirect effect, that is, the association between E-DII and depression risk is mediated by BMI.

In addition, to effectively understand the complex relationship between E-DII, PA and OA, we used a counterfactual mediation model that allows exposure of mediators to interact. According to this model, PA represents the relationship between E-DII and $\mathrm{OA}^{(26-28)}$.

All statistical analyses were performed using the software package $\mathrm{R}$ (http://www.R-project.org, The R Foundation). A two-tailed $P$-value of $<0.05$ was considered statistically significant.

\section{Results}

\section{Baseline characteristics}

A total of 1249 participants were eligible for this study (median (interquartile range, IQR) age, 72 (68-78) years; 701 (56.1\%) male), of which $401(32 \cdot 1 \%)$ cases of OA were identified based on self-report. The average E-DII in this study was +0.68 (SE 0.08), and the score ranged from -5.32 (most antiinflammatory) to +4.26 (most pro-inflammatory). Table 1 shows the distribution of characteristics across E-DII quartiles. Compared with subjects in the most anti-inflammatory E-DII category, the most pro-inflammatory subjects were more likely to be young, male, college degree or above, nonHispanic white, normal weight, had lower serum Ca and phosphorus concentrations $(P<0.05)$ (Table 1$)$.

\section{Energy-adjusted Dietary Inflammatory Index and osteoarthritis}

Table 2 shows the rough and adjusted logistic regression results, describing the odds of OA compared with non-OA. In the first two models, E-DII was positively associated with the risk of $\mathrm{OA}$ in the second quartile (OR: 1.16 (95\% CI: $1 \cdot 06,1.68)$ ) to the fourth quartile (OR: 1.64 (95\% CI: 1.13 , $2 \cdot 37)$ ) compared with the first quartile. The analysis using E-DII to add 1-SD produced similar results (model 2: OR: $1 \cdot 12$ (95\% CI: 1.05, 1.35)). The spline variable confirmed that E-DII was non-linearly related to the risk of OA $(P=0.012)$, and the graph shows that the increase in E-DII was accompanied by an increase in the risk of OA (as shown in Fig. 2). After including PA (model 3), all associations weakened and became invalid $(P>0.05)$ (Table 2$)$.

\section{Energy-adjusted Dietary Inflammatory Index and osteoarthritis risk stratified by physical activity category}

Table 3 shows the association between E-DII and OA stratified by quartile categories of PA. An interaction between E-DII and PA on the risk of OA was observed $\left(P_{\text {Interaction }}<0.001\right)$. When stratified by PA category, among people with low levels of $\mathrm{PA}$, the risk of E-DII and OA were significantly positively correlated (model 2: OR: $2 \cdot 12$ (95\% CI: $1 \cdot 46,3 \cdot 26)$, fourth quartile of E-DII $v$. the first quartile). At the same time, in people with high levels of PA, low anti-inflammatory levels showed a protective effect on OA (model 2: OR: 0.75 (95\% CI: 0.51, 0.90), the second quartile of E-DII $v$. the first quartile), and no significant association was observed in other populations.

\section{The mediating role of $\mathrm{BMI}$}

The result of the mediation analysis is shown in Fig. 3. First, we hypothesised a simple mediation model without the effect of the E-DII $\times$ PA interaction on the risk of OA. An increase in E-DII was associated with an increased risk of OA. This effect (22.82\%) could be explained by the significant indirect effect of PA (natural indirect effect: OR: 1.058 (95\% CI: 1.012, 1.126), as shown in Fig. 3(a)). Then, based on the aforementioned interaction of E-DII and PA on the risk of OA, we used a mediation model that 
NS British Journal of Nutrition

Table 1. Distribution of characteristics across quartiles of Dietary Inflammatory Index (DII) in National Health and Nutrition Examination Surveys, USA, 2011-2016* (Mean values and standard deviations; numbers and percentages)

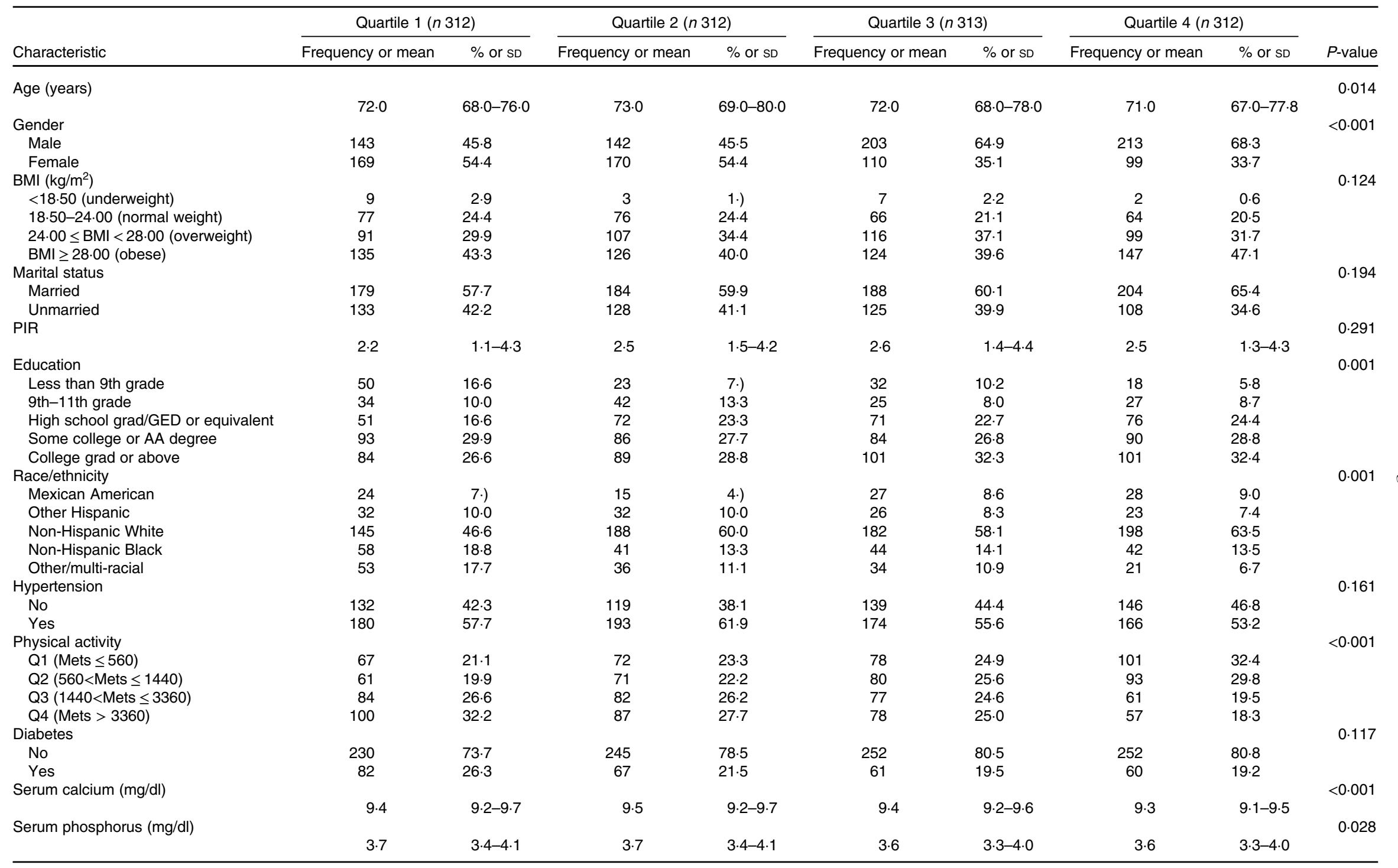

PIR, poverty:income ratio.

Quartile 1: -532 to -0 08; Quartile 2: -0 07, 1 22; Quartile 3: 123 to 2 26; Quartile 4: 227 to 426. 
Table 2. Risk of osteoarthritis according to quartile groups of Energy-Adjusted Dietary Inflammatory Index (E-DII) ( $n$ 1249)

\begin{tabular}{|c|c|c|c|c|c|c|c|c|c|c|}
\hline & \multicolumn{2}{|c|}{$\begin{array}{l}\text { Participants } \\
\text { without } O A\end{array}$} & \multicolumn{2}{|c|}{$\begin{array}{c}\text { Participants } \\
\text { with } \mathrm{OA}\end{array}$} & \multicolumn{2}{|c|}{ Model $1^{a}$} & \multicolumn{2}{|c|}{ Model $2^{\mathrm{b}}$} & \multicolumn{2}{|c|}{ Model $3^{c}$} \\
\hline & $n$ & $\%$ & $n$ & $\%$ & OR & $95 \% \mathrm{Cl}$ & OR & $95 \% \mathrm{Cl}$ & OR & $95 \% \mathrm{Cl}$ \\
\hline For 1-sD increase & 848 & & 401 & & 1.08 & $1.01,1 \cdot 17$ & $1 \cdot 12$ & $1.05,1.35$ & 1.06 & $0.95,1.28$ \\
\hline \multicolumn{11}{|c|}{ Quartile groups of E-DII } \\
\hline Q1 & 221 & $26 \cdot 1$ & 91 & $22 \cdot 7$ & Reference & & & & & \\
\hline Q2 & 207 & $24 \cdot 4$ & 105 & $26 \cdot 2$ & 1.04 & $0.74,1.47$ & $1 \cdot 16$ & $1.06,1.68$ & 1.15 & $0.81,1.66$ \\
\hline Q3 & 219 & $25 \cdot 8$ & 94 & 23.4 & 1.22 & $1.02,1.72$ & 1.25 & $1.12,1.80$ & 1.21 & $0.86,1.68$ \\
\hline Q4 & 201 & 23.7 & 111 & $27 \cdot 7$ & 1.34 & $1.08,1.88$ & 1.64 & $1 \cdot 13,2 \cdot 37$ & 1.46 & $0.92,1.92$ \\
\hline$P$-trend & & & & & 0.016 & & 0.002 & & 0.102 & \\
\hline
\end{tabular}

OA, osteoarthritis; PIR, poverty:income ratio; PA, physical activity.

a Model 1: unadjusted.

${ }^{\mathrm{b}}$ Model 2: age, sex, BMI, education level, race, hypertension, diabetes, marital status, PIR, serum Ca and phosphorus.

${ }^{\mathrm{c}}$ Model 3: further adjusted for PA.

(a)

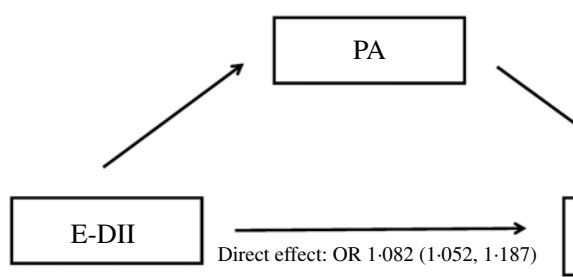

Effect

Controlled direct effect $=$ Natural direct effect

Natural direct effect

Total effect
Indirect effect: OR $1.058(1.012,1.126)$ Proportion mediated: $22.82 \%$

Estimate $^{\mathrm{a}}$
1.082
1.058
1.186

$1 \cdot 186$
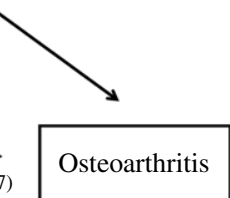

$95 \% \mathrm{Cl}^{\mathrm{b}}$

$1 \cdot 052,1 \cdot 187$

$1 \cdot 012,1 \cdot 126$

$1 \cdot 085,1 \cdot 402$ (b)

Indirect effect: OR 1.032 (1.016, 1.068) Proportion mediated: $20 \cdot 08 \%$

Fig. 3. Mediation analyses without (a) and with (b) an interaction between Energy-Adjusted Dietary Inflammatory Index (E-DII) and physical activity (PA) on the risk of osteoarthritis (OA). (a) Logistic model adjusted for age, sex, BMI, education level, race, hypertension, diabetes, marital status, poverty:income ratio (PIR), serum calcium and phosphorus. (b) The $95 \% \mathrm{Cl}$ of these estimates was computed using the bootstrap method (1000 samples).

allows exposure-media interaction. According to this model, PA was both a mediating factor and an influencing factor in the relationship between E-DII and OA. An increase in E-DII was also associated with an increased risk of OA (natural indirect effect and natural indirect effect OR: 1.056 (95\% CI: 1.012, 1.092) and OR: 1.032 (95\% CI: 1.016, 1.068)), but the proportion explained by the indirect influence of PA was low $(20.08 \%$, shown in Fig. 3(b)).

\section{Discussion}

On the basis of the baseline data of 1249 elderly people in the NHANES, we found a positive and nonlinear relationship between the baseline inflammation characteristics of diets and the risk of $\mathrm{OA}$ and that this relationship was independent of most known or potential risk factors or confounding factors. Participants with a higher DII (corresponding to the increased proinflammatory potential of diets) had a higher risk of OA than participants with a lower DII (corresponding to anti-inflammatory diets). This association was partly mediated by PA and its interaction with DII.
Observational studies on single dietary ingredients have not been confirmed through randomised trials. For example, compared with placebos, vitamin E supplements are ineffective, and vitamin D does not have a clinically important effect on OA joint pain ${ }^{(29)}$. However, people do not consume nutrients or food in isolation. In the past few years, people have increasingly emphasised the importance of diet as a whole ${ }^{(30,31)}$. Using the dietary index to quantify the inflammatory potential of the entire diet may increase the robustness and effectiveness of detecting the relationship between diseases ${ }^{(30)}$. DII and logically expanded E-DII explain the relationship between various nutrients and foods and their underlying inflammation, and evidence showing that these nutrients and foods exert a proinflammatory or anti-inflammatory effect in diets exists ${ }^{(21)}$. Our research is an improvement on previous observational studies that focused on single dietary components. This is a study that used DII to evaluate the relationship between the inflammatory potential of diets and OA. Our findings, if demonstrated in other large-scale prospective studies, may provide information for the development of prevention strategies with increased emphasis on the overall effect of diets for this highly prevalent debilitating disease. 
Table 3. Risk of osteoarthritis by quartile of dietary inflammatory index stratified according to physical activity (Numbers and percentages, $n$ 1249)

\begin{tabular}{|c|c|c|c|c|c|c|c|c|c|}
\hline & \multicolumn{2}{|c|}{$\begin{array}{c}\text { Participants } \\
\text { without OA }\end{array}$} & \multicolumn{2}{|c|}{$\begin{array}{l}\text { Participants } \\
\text { with OA }\end{array}$} & \multicolumn{2}{|c|}{ Model $1^{a}$} & \multicolumn{2}{|c|}{ Model $2^{b}$} & \multirow[b]{2}{*}{$P_{\text {Interaction }}$} \\
\hline & $N$ & $\%$ & $n$ & $\%$ & OR & $95 \% \mathrm{Cl}$ & OR & $95 \% \mathrm{Cl}$ & \\
\hline Mets $\leq 560(n 318)$ & & & & & & & & & $<0.001$ \\
\hline For 1-SD increase & $n 214$ & & $n 104$ & & 1.13 & $1.01,1.26$ & 1.24 & $1.09,1.42$ & \\
\hline Q1 & 56 & $26 \cdot 2$ & 21 & $20 \cdot 2$ & Reference & & Reference & & \\
\hline Q2 & 61 & 28.5 & 33 & 31.7 & 1.08 & $0.86,1.92$ & 1.59 & $1 \cdot 25,3 \cdot 16$ & \\
\hline Q3 & 59 & $27 \cdot 6$ & 21 & $20 \cdot 2$ & 1.44 & $0.92,2.78$ & $1 \cdot 82$ & $1.50,2.61$ & \\
\hline Q4 & 38 & $17 \cdot 8$ & 29 & $27 \cdot 9$ & 2.04 & $1.02,3.08$ & $2 \cdot 12$ & $1 \cdot 46,3 \cdot 26$ & \\
\hline \multicolumn{10}{|c|}{$560<$ Mets $\leq 1440(n 305)$} \\
\hline For 1-SD increase & $n 200$ & & $n 105$ & & 1.02 & $0.91,1.13$ & 1.05 & $0.93,1.19$ & \\
\hline Q1 & 59 & $29 \cdot 5$ & 26 & $24 \cdot 8$ & Reference & & Reference & & \\
\hline Q2 & 49 & 24.5 & 25 & $23 \cdot 8$ & $1 \cdot 16$ & $0.59,2.26$ & 1.09 & $0.76,2 \cdot 26$ & \\
\hline Q3 & 42 & $21 \cdot 0$ & 25 & $23 \cdot 8$ & $1 \cdot 32$ & $0.68,2.52$ & 1.64 & $0.85,3.48$ & \\
\hline Q4 & 50 & $25 \cdot 0$ & 29 & $27 \cdot 6$ & 1.42 & $0.72,2.66$ & 1.65 & $0.87,3.39$ & \\
\hline \multicolumn{10}{|c|}{$1440<$ Mets $\leq 3360(n 304)$} \\
\hline For 1-sD increase & $n 201$ & & $n 103$ & & 0.97 & $0.87,1.08$ & 0.97 & $0.86,1.10$ & \\
\hline Q1 & 52 & $25 \cdot 9$ & 29 & $28 \cdot 2$ & Reference & & Reference & & \\
\hline Q2 & 50 & 24.9 & 25 & $24 \cdot 3$ & 0.90 & $0.46,1.74$ & 0.68 & $0.33,1.40$ & \\
\hline Q3 & 50 & $24 \cdot 9$ & 22 & 21.4 & 0.79 & $0.40,1.74$ & 0.93 & $0.44,1.98$ & \\
\hline Q4 & 49 & 24.4 & 27 & $26 \cdot 2$ & 0.99 & $0.51,1.90$ & 1.02 & $0.49,2.15$ & \\
\hline \multicolumn{10}{|l|}{ Mets > $3360(n$ 322) } \\
\hline For 1-SD increase & $n 233$ & & $n 89$ & & 0.92 & $0.72,1.08$ & 0.86 & $0.58,0.98$ & \\
\hline Q1 & 54 & $23 \cdot 2$ & 15 & $16 \cdot 9$ & Reference & & Reference & & \\
\hline Q2 & 47 & $20 \cdot 2$ & 22 & $24 \cdot 7$ & 0.82 & $0.62,0.98$ & 0.75 & $0.51,0.90$ & \\
\hline Q3 & 68 & $29 \cdot 2$ & 26 & $29 \cdot 2$ & 0.96 & $0.75,1.16$ & 0.88 & $0.62,0.96$ & \\
\hline Q4 & 64 & $27 \cdot 5$ & 26 & $29 \cdot 2$ & 1.25 & $0.70,2.85$ & 1.08 & $0.75,1.82$ & \\
\hline
\end{tabular}

$\mathrm{OA}$, osteoarthritis; Q, quartile; PIR, poverty:income ratio; E-DII, Energy-adjusted Dietary Inflammatory Index; PA, physical activity.

a Model 1: unadjusted.

${ }^{b}$ Model 2: age, sex, BMI, education level, race, hypertension, diabetes, marital status, PIR, serum Ca and phosphorus.

${ }^{\mathrm{c}} P_{\text {Interaction }}$ was calculated using the multiplicative interaction term (E-DII $\times$ PA).

A large number of studies have shown that DII is significantly related to serum inflammation markers ${ }^{(32-35)}$. Therefore, the site of OA inflammation can be assessed early before a considerable radiological change (synovitis) ${ }^{(36)}$. These findings have also been confirmed by some studies using MRI ${ }^{(37,38)}$. Therefore, chronic low-grade inflammation may be the main driver of persistent bone degeneration. Our findings indirectly confirmed the role of inflammation in the prediction of OA stage. Second, changes in the extracellular matrix may play an additional role in the association between DII and OA. Extracellular matrix breakdown is common in areas of inflammation, including bones affected by $\mathrm{OA}^{(39)}$. In addition, studies have demonstrated that extracellular matrix breakdown products themselves can promote inflammation and cartilage loss ${ }^{(40)}$. Therefore, proinflammatory diets may further promote the process of cartilage loss. Finally, considering that individuals with significantly higher DII levels ate meat, sugar and fat more frequently and consumed fewer vegetables and fruits than those with lower DII levels, DII can be assumed to be negatively correlated with healthy eating patterns (such as the Mediterranean diet), which may play a role in protecting people from OA attacks.

To the best of our knowledge, this is the first study to show that weekly moderate/vigorous exercise is an important part of the mediation of the relationship between systemic low-grade dietary inflammation and the subsequent increased risk of OA. The consistency of this finding with previous results indicated that PA had a central role in bone health and the regulation of peripheral inflammatory processes ${ }^{(41,42)}$. Routine PA has been associated with a reduced risk of OA in people aged 50 years and older. Moreover, previous studies have revealed a correlation between systemic inflammation and PA. In this study, low PA was found to be significantly associated with an increased risk of OA. In addition, elevated DII was significantly associated with low $\mathrm{PA}^{(43)}$. Therefore, our results indicated that individuals with elevated levels of systemic inflammation may be less likely to exercise moderately or vigorously every week than those without. This situation may increase the risk of experiencing elevated levels of OA. Importantly, PA explained approximately one-fifth of the total effect of systemic dietary inflammation on the subsequent elevation of OA. Future research needs to identify other intermediate mechanisms that explain this relationship. Our findings indicated that conventional PA may not only be a valuable tool for preventing OA but may also have potential antiinflammatory effects.

The exact biological mechanism that links the inflammatory process to OA through the action of PA is not fully understood. However, systemic low-grade inflammation and PA have been suggested to act on the same effective system of the body: the hypothalamic-pituitary-adrenal axis and the sympathetic nervous system ${ }^{(44)}$. For example, elevated levels of proinflammatory cytokines are repeatedly associated with the overactivation of the hypothalamic-pituitary-adrenal axis, the excessive secretion of stress hormones (such as cortisol), the impaired production of serotonin and dopamine and the initiation of oxidative stress. In 
addition, regular PA can increase the body's serotonin synthesis, improve noradrenergic neurotransmission, trigger the release of endorphins and reduce long-term increased sympathetic nervous system activity ${ }^{(45,46)}$. PA is also related to the increase in muscle-derived IL- 6 . In this case, IL- 6 can act as an anti-inflammatory muscle factor by inhibiting TNF- $\alpha$. Importantly, these shared biological mechanisms play a major role in the pathophysiology of $\mathrm{OA}^{(47,48)}$.

Our research still has some limitations. First of all, the main limitation of the current design is that NHANES is a cross-sectional database and thus severely hindered our ability to perform causal reasoning. Second, dietary intake was self-reported, with recall bias, estimated on the basis of a 24-h history, and could not reflect changes in daily dietary intake. Among the forty-five possible food parameters, only twenty-eight can be used for the calculation of DII, and previous studies have successfully verified the modified DII (twenty-eight items) in NHANES. However, these unbalanced twenty-seven items (if they are not equally divided into proinflammatory and anti-inflammatory item) may also erroneously distort the inflammatory potential of diets. Third, the measurement of PA was based on self-reporting rather than objective methods, and the risk of self-reporting bias was present. Finally, the judgements of study participants' OA, diabetes and hypertension were based on self-report, and there is no clinical diagnosis.

\section{Conclusion}

In summary, our findings indicated that a diet with high proinflammatory potential was associated with a higher risk of $\mathrm{OA}$ in the elderly, and PA was one of the main mediators that may occur here. Our findings may provide some evidence for understanding the underlying mechanisms of dietary inflammation, PA and OA. Considering the cross-sectional design of NHANES, it is necessary to conduct further prospective cohort studies.

\section{Acknowledgements}

The present study used data from the NHANES. We thank all of the participants and staff involved in the surveys.

The project was supported by the National Natural Science Foundation of China (Grant Nos. 81974339 and 81873988) and the Science and Technology Plan Project of Hunan Province (Grant No. 2021GK2012). The funders did not play any role in the study design, data collection and analysis, decision to publish or preparation of the manuscript.

Y. H., J. X. and H. W. had full access to all of the data in the study and take responsibility for the integrity of the data and the accuracy of the data analysis. Concept and design: Y. H., J. X. and $\mathrm{H}$. W. Acquisition, analysis or interpretation of data: All authors. Drafting of the manuscript: Y. H., J. X., H. W. and R. L. Critical revision of the manuscript for important intellectual content: All authors. Statistical analysis: H. W., R. L., W. T., W. S. and M. Z. Obtained funding: Y. H. and J. X. Administrative, technical or material support: H. W., R. L., J. Y. and X. F. Supervision: Y. H.

There are no conflicts of interest.

\section{References}

1. Park J, Mendy A \& Vieira Edgar R (2018) Various types of arthritis in the United States: prevalence and age-related trends from 1999 to 2014. Am J Public Health 108, 256-258.

2. Conaghan PG, Porcheret M, Kingsbury SR, et al. (2015) Impact and therapy of OA: the arthritis care OA nation 2012 survey. Clin Rheumatol 34, 1581-1588.

3. Hackney Alisha J, Jennifer KN, Resnick B, et al. (2019) Association of systemic inflammation and fatigue in OA: 2007-2010 national health and nutrition examination survey. Biol Res Nurs 21, 532-543.

4. Damman W, Liu R, Reijnierse M, et al. (2021) Effusion attenuates the effect of synovitis on radiographic progression in patients with hand OA: a longitudinal magnetic resonance imaging study. Clin Rheumatol 40, 315-319.

5. Kondo F, Takegami Y, Ishizuka S, et al. (2021) The association of the progression of knee OA with high-sensitivity CRP in community-dwelling people - the Yakumo study. Clin Rheumatol 40, 2643-2649.

6. Shadyab AH, Terkeltaub R, Kooperberg C, et al. (2018) Prospective associations of C-reactive protein (CRP) levels and CRP genetic risk scores with risk of total knee and hip replacement for $\mathrm{OA}$ in a diverse cohort. $\mathrm{OA}$ Cartilage 26, 1038-1044.

7. Sakellariou G, Quaglini S, Bugatti S, et al. (2021) In patients with early peripheral psoriatic arthritis baseline C-reactive protein, pain and ultrasound-detected synovitis predict subsequent treatment with ts/bDMARDs. A retrospective analysis. J Clin Med 10, 2834.

8. Yang X, Ruan G, Xu J, et al. (2020) Associations between suprapatellar pouch effusion-synovitis, serum cartilage oligomeric matrix protein, high sensitivity C-reaction protein, knee symptom, and joint structural changes in patients with knee osteoarthritis. Clin Rheumatol 39, 1663-1670.

9. Li R, Zhan W, Huang X, et al. (2021) Association of dietary inflammatory index (DII) and metabolic syndrome in the elderly over 55 years in Northern China. Br J Nutr 18, 1-8.

10. Wood LG, Shivappa N, Berthon BS, et al. (2015) Dietary inflammatory index is related to asthma risk, lung function and systemic inflammation in asthma. Clin Exp Allergy 45, 177-183.

11. Hammad Shatha S, Mahmoud R, Shivappa N, et al. (2021) Dietary inflammatory index and odds of breast cancer: a case-control study. Food Sci Nutr 9, 5211-5219.

12. Abulimiti A, Zhang X, Shivappa N, et al. (2020) The dietary inflammatory index is positively associated with colorectal cancer risk in a Chinese case-control study. Nutrients 12, 232.

13. Morimoto M, Shivappa N, de Souza Genaro P, et al. (2019) Lack of association between dietary inflammatory index and low impact fractures in the Brazilian population: the Brazilian osteoporosis study (BRAZOS). Adv Rheumatol 59, 16.

14. Skou ST, Bricca A \& Roos EM (2018) The impact of PA level on the short- and long-term pain relief from supervised exercise therapy and education: a study of 12,796 Danish patients with knee OA. OA Cartilage 26, 1474-1478.

15. Draganidis D, Jamurtas Athanasios Z, Stampoulis T, et al. (2018) Disparate Habitual physical activity and dietary intake profiles of elderly men with low and elevated systemic inflammation. Nutrients 10, 566.

16. Joseph Kenth L, Dagfinrud H, Christie A, et al. (2021) Criterion validity of the international PA questionnaire-short form (IPAQ$\mathrm{SF}$ ) for use in clinical practice in patients with OA. $B M C$ Musculoskelet Disord 22, 232.

17. Liao C-D, Liao Y-H, Liou T-H, et al. (2021) Effects of protein-rich nutritional composition supplementation on sarcopenia indices and PA during resistance exercise training in older women with knee OA. Nutrients 13, 2487. 
18. National Center for Health Statistics (2013) National Health and Nutrition Examination Survey, 2013-2014 Overview. https:// www.cdc.gov/nchs/data/nhanes/nhanes_13_14/2013-14_over view_brochure.pdf (accessed February 2022).

19. Shivappa N, Steck SE, Hurley TG, et al. (2014) Designing and developing a literature-derived, population-based dietary inflammatory index. Public Health Nutr 17, 1689-1696.

20. Tabung FK, Smith-Warner SA, Chavarro JE, et al. (2016) Development and validation of an empirical dietary inflammatory index. J Nutr 146, 1560-1570.

21. Hallal PC, Andersen LB, Bull FC, et al. (2012) Global PA levels: surveillance progress, pitfalls, and prospects. Lancet $\mathbf{3 8 0}$ 247-257.

22. National Health and Nutrition Examination Survey 2015-2016 Data Documentation, Codebook, and Frequencies. https:// wwwn.cdc.gov/Nchs/Nhanes/2015-2016/PAQ_I.htm (accessed February 2022).

23. Loprinzi Paul D (2015) Dose-response association of moderate-to-vigorous physical activity with cardiovascular biomarkers and all-cause mortality: considerations by individual sports, exercise and recreational physical activities. Prev Med 81, 73-77.

24. Loeser RF, Beavers DP, Bay-Jensen AC, et al. (2017) Effects of dietary weight loss with and without exercise on interstitial matrix turnover and tissue inflammation biomarkers in adults with knee osteoarthritis: the intensive diet and exercise for arthritis trial (IDEA). OA Cartilage 25, 1822-1828.

25. Veronese N, Shivappa N, Stubbs B, et al. (2019) The relationship between the dietary inflammatory index and prevalence of radiographic symptomatic osteoarthritis: data from the osteoarthritis initiative. Eur J Nutr 58, 253-260.

26. Valeri L \& Vanderweele TJ (2013) Mediation analysis allowing for exposure mediator interactions and causal interpretation: theoretical assumptions and implementation with SAS and SPSS macros. Psychol Methods 18, 137-150.

27. Arfan IM \& VanderWeele TJ (2015) A proposed clinical and biological interpretation of mediated interaction. Eur J Epidemiol 30, $1115-1118$

28. Böhnke Jan R (2016) Explanation in causal inference: methods for mediation and interaction. QJ Exp Psychol 69, 1243-1244.

29. Liu X, Machado Gustavo C, Eyles Jillian P, et al. (2018) Dietary supplements for treating OA: a systematic review and metaanalysis. Br J Sports Med 52, 167-175.

30. Hu Frank B (2002) Dietary pattern analysis: a new direction in nutritional epidemiology. Curr Opin Lipidol 13, 3-9.

31. Sacks FM, Obarzanek E, Windhauser MM, et al. (1995) Rationale and design of the dietary approaches to stop hypertension trial (DASH). A multicenter controlled-feeding study of dietary patterns to lower blood pressure. Ann Epidemiol 5, 108-118.

32. Cavicchia PP, Steck SE, Hurley TG, et al. (2009) A new dietary inflammatory index predicts interval changes in serum highsensitivity C-reactive protein. J Nutr 139, 2365-2372.

33. Crimarco A, Turner-McGrievy GM, Wirth MD, et al. (2019) Baseline markers of inflammation, lipids, glucose, and dietary inflammatory index scores do not differ between adults willing to participate in an intensive inflammation reduction intervention and those who do not. Nutr Health 25, 9-19.

34. Shin P-K, Park S-J, Kim Myung S, et al. (2020) A traditional Korean diet with a low dietary inflammatory index increases anti-inflammatory IL-10 and decreases pro-inflammatory NF$\kappa \mathrm{B}$ in a small dietary intervention study. Nutrients 12, 2468.

35. Kaluza J, Harris H, Melhus H, et al. (2018) Questionnaire-based anti-inflammatory diet index as a predictor of low-grade systemic inflammation. Antioxid Redox Signal 28, 78-84.

36. Sokolove J \& Lepus CM (2013) Role of inflammation in the pathogenesis of OA: latest findings and interpretations. Therapeutic Adv Musculoskeletal Dis 5, 77-94.

37. Felson David T, McLaughlin S, Goggins J, et al. (2003) Bone marrow edema and its relation to progression of knee OA. Ann Intern Med 139, 330-336.

38. Krasnokutsky S, Belitskaya-Lévy I, Bencardino J, et al. (2011) Quantitative magnetic resonance imaging evidence of synovial proliferation is associated with radiographic severity of knee OA. Arthritis Rheum 63, 2983-2991.

39. Krasnokutsky S, Belitskaya-Lévy I, Bencardino J, et al. (2011) Quantitative magnetic resonance imaging evidence of synovial proliferation is associated with radiographic severity of knee OA. Arthritis Rheum 63, 2983-2991.

40. Homandberg GA \& Hui F (1996) Association of proteoglycan degradation with catabolic cytokine and stromelysin release from cartilage cultured with fibronectin fragments. Arch Biochem Biophys 334, 325-331.

41. Cleland Brice T, Papanek P, Ingraham Benjamin A, et al. (2020) Determinants of low bone mineral density in people with multiple sclerosis: role of PA. Mult Scler Relat Disord 38, 101864.

42. Tomlinson David J, Erskine Robert M, Morse Christopher I, et al. (2019) Body fat percentage, body mass index, fat mass index and the ageing bone: their singular and combined roles linked to PA and diet. Nutrients 11, 195.

43. Hamaguchi K, Kurihara T, Fujimoto M, et al. (2020) Associations among bone mineral density, PA and nutritional intake in middle-aged women with high levels of arterial stiffness: a pilot study. Int J Environ Res Public Health 17, 1620.

44. Cotman Carl W, Berchtold Nicole C \& Christie L-A (2007) Exercise builds brain health: key roles of growth factor cascades and inflammation. Trends Neurosci 30, 464-472.

45. Picke A-K, Sylow L, Møller Lisbeth LV, et al. (2018) Differential effects of high-fat diet and exercise training on bone and energy metabolism. Bone 116, 120-134.

46. Kawao N, Iemura S, Kawaguchi M, et al. (2021) Role of irisin in effects of chronic exercise on muscle and bone in ovariectomized mice. J Bone Miner Metab 39, 547-557.

47. Bressi B, Cagliari M, Contesini M, et al. (2021) Physical exercise for bone health in men with prostate cancer receiving androgen deprivation therapy: a systematic review. Support Care Cancer 29, 1811-1824.

48. Qi Z, Liu W \& Lu J (2016) The mechanisms underlying the beneficial effects of exercise on bone remodeling: roles of bonederived cytokines and microRNAs. Prog Biophys $\mathrm{Mol}$ Biol 122, 131-139. 